\title{
Animal Assisted Therapy in Pediatric Speech-Language Therapy with a Preschool Child with Severe Language Delay: A Single- Subject Design
}

\author{
Heather K. Anderson \\ Louisiana State University Health Sciences Center - Shreveport, hande1@lsuhsc.edu \\ Sandra L. Hayes \\ Louisiana State University Health Sciences Center Shreveport, shayes1@lsuhsc.edu \\ Julie P. Smith \\ Louisiana State University Health Sciences Center Shreveport, jsmi53@lsuhsc.edu
}

Follow this and additional works at: https://nsuworks.nova.edu/ijahsp

Part of the Medicine and Health Sciences Commons

\section{Recommended Citation}

Anderson HK, Hayes SL, Smith JP. Animal Assisted Therapy in Pediatric Speech-Language Therapy with a Preschool Child with Severe Language Delay: A Single-Subject Design. The Internet Journal of Allied Health Sciences and Practice. 2019 Jan 01;17(3), Article 1.

This Manuscript is brought to you for free and open access by the College of Health Care Sciences at NSUWorks. It has been accepted for inclusion in Internet Journal of Allied Health Sciences and Practice by an authorized editor of NSUWorks. For more information, please contact nsuworks@nova.edu. 


\title{
Animal Assisted Therapy in Pediatric Speech-Language Therapy with a Preschool Child with Severe Language Delay: A Single-Subject Design
}

\begin{abstract}
Background: The application of animal assisted therapy (AAT) in provision of services is an emerging area of research in the allied health literature. Prior investigators have called for additional research concerning applications of animal assisted therapy in specific settings and patient populations.

Objectives: to (a) investigate the effect of animal assisted therapy on the quantity of vocalizations in a single child participant with severe speech delay, and (b) identify optimal animal assisted therapy practices in pediatric group speech-language therapy.

Design: A case study was conducted using ABA single-case design. The number of vocalizations produced by the participant was measured for 15-minute periods during four initial baseline (no animal assisted therapy) sessions, four sessions with the intervention condition (animal assisted therapy), and three additional baseline (no animal assisted therapy) sessions. Observations were also recorded concerning the interactions between the animal assisted therapy team, the participant, and other children in the group.

Results: The number of vocalizations increased markedly during the intervention phase, and the effect was nonreversible. The participant also demonstrated increased attention to tasks and activities during the intervention phase. An increase in unpredictable, forceful movements by the participant and other children was observed after 10-minutes.
\end{abstract}

Conclusions: Although the same degree of increase in vocalizations is not expected for every child exposed to animal assisted therapy, results suggest that animal assisted therapy is a potentially valuable tool for speech-language pathologists working with children who have severe delays in communication skills. Recommendations for future research include consideration of time limits for animal assisted therapy interventions, detailed advance planning with the handler to minimize stressors for the animal assisted therapy team, and ensuring adequate adult personnel for data collection and management of the intervention sessions.

\section{Author Bio(s)}

Heather Anderson, MA, CCC-SLP, is an Assistant Professor of Clinical Communication Disorders in the School of Allied Health Professions at Louisiana State University Health Sciences Center-Shreveport. She is a licensed and certified speech-language pathologist, and is also a doctoral candidate in the EdD program at Louisiana Tech University, College of Education.

Sandra Hayes, SLP.D., MCD, CCC-SLP, is Program Director and Associate Professor of Clinical Communication Disorders in the Speech-Language Pathology Program in the School of Allied Health Professions at Louisiana State University Health Sciences Center-Shreveport. She is a licensed and certified speech-language pathologist. Dr. Hayes and Boomer Hayes are a certified animal assisted therapy team.

Julie Smith, MA, CCC-SLP, is an Instructor of Clinical Communication Disorders in the Speech-Language Pathology Program in the School of Allied Health Professions at Louisiana State University Health Sciences Center-Shreveport. She is a licensed and certified speech-language pathologist. 


\title{
1JAHSP \\ The Internet Joutnal of Allied Health Sciences and Practice
}

Dedicated to allied health professional practice and education

Vol. 17 No. 3 ISSN 1540-580X

\section{Animal-Assisted Therapy in Pediatric Speech-Language with a Preschool Child with Severe Language Delay: A Single-Subject Design}

\author{
Heather K. Anderson \\ Sandra L. Hayes \\ Julie P. Smith \\ Louisiana State University \\ United States
}

\begin{abstract}
Background: The application of animal assisted therapy (AAT) in provision of services is an emerging area of research in the allied health literature. Prior investigators have called for additional research concerning applications of animal assisted therapy in specific settings and patient populations. Objectives: The objectives of this study are twofold: a) to investigate the effect of animal assisted therapy on the quantity of vocalizations in a single child participant with severe speech delay, and b) to identify optimal animal assisted therapy practices in pediatric group speech-language therapy. Design: A case study was conducted using ABA single-case design. The number of vocalizations produced by the participant was measured for 15-minute periods during four initial baseline (no animal assisted therapy) sessions, four sessions with the intervention condition (animal assisted therapy), and three additional baseline (no animal assisted therapy) sessions. Observations were also recorded concerning the interactions between the animal assisted therapy team, the participant, and other children in the group. Results: The number of vocalizations increased markedly during the intervention phase, and the effect was nonreversible. The participant also demonstrated increased attention to tasks and activities during the intervention phase. An increase in unpredictable, forceful movements by the participant and other children was observed after 10-minutes. Conclusions: Although the same degree of increase in vocalizations is not expected for every child exposed to animal assisted therapy, results suggest that animal assisted therapy is a potentially valuable tool for speech-language pathologists working with children who have severe delays in communication skills. Recommendations for future research include consideration of time limits for animal assisted therapy interventions, detailed advance planning with the handler to minimize stressors for the animal assisted therapy team, and ensuring adequate adult personnel for data collection and management of the intervention sessions.
\end{abstract}

Keywords: animal assisted therapy (AAT), language delay, pediatric language, speech therapy, service animal welfare 


\section{INTRODUCTION}

Animal assisted therapy (AAT) has a potential tool to enhance the effectiveness of educational and rehabilitation programs for young children and individuals with disabilities, including speech and language disorders. One of the most common goals incorporated in treatment programs for young children with speech and language delays is to increase the frequency and complexity of verbal output. In cases of severe speech/language delays, when children have no intelligible words, the initial goal may be to facilitate increased attempts at speech and encourage vocalizations of any kind. Animal assisted therapy can be incorporated into the intervention by bringing an AAT team into group and individual sessions as a means of facilitating additional client vocalizations and/or verbalizations and increasing participation in therapy activities.

This study was conducted at a university-affiliated clinic that serves a diverse population of individuals with communication disorders while also providing clinical education opportunities for master's level speech-language pathology students enrolled in the university. The purpose of this case study research project was twofold. First, the case study was designed to investigate whether the number of spontaneous vocalizations produced by a 3-year-old child, who was enrolled in a group speech/language therapy program, increased during the time the AAT team was involved in the session. The hypothesis was that when AAT was introduced, the number of spontaneous vocalizations (defined as an instance of phonation perceptually within the same pitch and loudness range expected for speech production, including unintelligible utterances, exclamations [e.g., ooh, hey], word approximations, or words, produced spontaneously or imitated spontaneously [i.e., without adult direction to imitate the sound/word]) produced by the child during 15-minute intervals would be greater than the number of vocalizations produced per 15minute intervals in sessions without AAT. Second, the case study was designed to identify successful practices and potential barriers to conducting research involving AAT, so that the findings could be applied to develop future AAT research with children and adults.

\section{LITERATURE REVIEW}

Research involving AAT has employed a variety of research questions, participant characteristics, and methods. Animal assisted therapy has been found to impact a broad range of behaviors. ${ }^{1}$ Outcomes have included increased attending skills and cooperative behaviors, increased eye contact, increased communicative exchanges with clinicians and peers, and fewer instances of tantrums, distractions, and inappropriate topic exchanges..$^{2-6}$ Investigators have also addressed the use of AAT with adults and children with different types of disabilities, such as emotional disturbances, multiple disabilities, Down syndrome, and children with varying severe disabilities..$^{2-4,7}$

In addition, AAT has also been investigated in regard to impact on different factors related to general well-being and psychosocial status. While Cole et al found no differences or positive effects of AAT on behaviors such as depression, hostility or anxiety, Motomura et al reported decreased levels of apathy among participants. ${ }^{8,9}$ As another example, Edwards and Beck documented increased nutritional intake for patients in extended-care facilities following the introduction of fish aquariums in dining areas. ${ }^{10}$

O'Haire identified increased social interaction as the most commonly reported outcome among studies of AAT with autism spectrum disorder (ASD). ${ }^{11}$ Sams et al investigation of 22 children with ASD found that introduction of AAT resulted in more frequent social interactions and use of language, with a larger effect for social interactions, during occupational therapy (OT) sessions. ${ }^{12}$ Sams et al concluded that these results were consistent with a "biocentric" view of child development, which holds that children naturally gravitate toward animals and other nonhuman elements in the environment. ${ }^{12(p .269)}$

Other researchers have investigated the effects of incorporating AAT into habilitative and/or rehabilitative therapy. For example, Velde et al conducted a qualitative investigation of how occupational therapists (OT's) and long-term care residents perceived their experiences with AAT. ${ }^{13}$ Results supported the use of AAT as a therapeutic modality by OT's, and the investigators identified applications of AAT for physical therapists (PT) and therapeutic recreation specialists (RT). In addition to OT, PT, and RT, investigators have also addressed AAT applications for speech-language pathology. Macauley and Gutierrez found increased motivation to attend speech-language therapy among child participants when AAT was used in speech-language therapy. ${ }^{14}$ Macauley also indicated that AAT was associated with increased satisfaction with speech-language therapy services among adults with neurogenic communication disorders. ${ }^{5}$

A key concern in any research involving therapy with service animals is the welfare of the animal(s). The majority of studies included in this review involved the use of dogs; however, other animals, such as fish, dolphins, and horses, have also been utilized.7,14 Endenburgh and van Lith noted that systematic research concerning threats to the welfare of therapy and service animals is currently lacking, but did cite multiple studies ${ }^{15-18}$ suggesting that factors in the environment or working condition may elevate the stress levels of dogs and other AAT animals. Burrows et al in a qualitative investigation of the use of therapy dogs in intervention programs for children with ASD, stressed the need to balance the welfare of the animal with the welfare of the child participants. ${ }^{19}$ 
In addition to concerns regarding the dogs themselves, stress placed on therapy dogs may also negatively impact the intervention, because stress could influence the dog's behavior in a manner that leads the dog to decrease interactions with participants. Multiple investigators have stressed the importance of directly addressing the welfare of service and therapy animals in future research.1,15,19

The literature on AAT research includes several studies that are particularly relevant to the primary purpose of the current case study, examining the effect of AAT on vocalizations in a child with severe language impairment. One study included children who exhibited impairments solely in the area of language, while other studies included children with impairments in more than one developmental domain. ${ }^{14}$ Results suggested that AAT was effective toward meeting speech, language, and motor goals, and

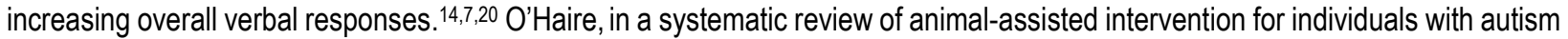
spectrum disorder (ASD), identified an overall positive trend in the effects of AAT on outcomes involving communication and/or use of language..$^{11}$

Although the research described above ${ }^{1,15-19}$ illustrates the emergence of scholarship addressing the establishment of optimal AAT practices and the impact of AAT on human communication behaviors, additional research is needed in both areas. Nimer and Lundahl conducted a meta-analysis of AAT studies that revealed positive outcomes associated with AAT across a variety of study designs, participant types, and sample sizes. ${ }^{1}$ However, in their study, Nimer and Lundahl noted a gap in the literature concerning optimal AAC practices for specific populations or settings; this gap was directly addressed by the present case study.

\section{METHODOLOGY}

This case study investigated the effect of animal assisted therapy (AAT) on the quantity of spontaneous vocalizations produced by a single male child, BD (pseudonym), age three years, one month. For the purpose of this case study, a spontaneous vocalization was defined by the authors as an instance of phonation perceptually within the same pitch and loudness range expected for speech production which, based on context, was produced in response to an identifiable stimulus and could be characterized as at least one of the following: unintelligible utterance, exclamations (e.g., ooh, hey), word approximation, or word, produced spontaneously (i.e., produced without a model) or imitated spontaneously (i.e., imitated by the child in the absence of adult direction to imitate the sound/word). Because the purpose of introducing AAT with BD was to attempt to diversify vocal behaviors and to establish conditions where vocal behaviors occurred as part of a clear stimulus-response pattern, certain noises were excluded from the operational definition of spontaneous vocalizations. Noises associated with crying, tantrums, or screaming, were excluded for two reasons: a) these behaviors had been observed during previous sessions, and the intervention was introduced in an attempt to elicit behaviors not previously observed; and $b$ ) such behaviors are more reflexive in nature and are characteristic of an earlier stage of pre-linguistic development, compared to more organized vocalizations produced in response to interactions or other stimuli. ${ }^{21}$

Similarly, vegetative noises, such as those produced during coughing or sneezing, were excluded because these behaviors do not reflect communicative intent or vocal play. Utterances produced imitatively on request (e.g., "Say train,") were also excluded, per the above definition of spontaneous vocalization. The number of vocalizations was selected as the dependent variable, rather than the number of verbalizations, because of the severity of BD's communication delay and prior lack of vocalizations, as noted in both clinical records and baseline data (A condition). The setting for the case study was a language therapy group for late talkers, in which BD was enrolled. The group was comprised of seven children, ranging in age from 2 years, 4 months through 3 years, 1 month. The children attended semiweekly sessions led by four graduate students and two clinical supervisors in a play environment at a university-affiliated speech, language and hearing clinic.

\section{Participant}

BD had previously been identified with developmental delays in speech/language skills and cognitive/play skills and referred for early intervention services. At the time of this case study, he had been enrolled in language therapy at this facility for approximately seven months. BD was chosen for this case study because prior to the initiation of the case study, he had been observed to be nonverbal during the group sessions. During sessions prior to the case study, as during the baseline (A) phase of the case study, he had not produced any recognizable words and had rarely produced vocalizations of any kind in the presence of the investigators or others involved in his care at the facility. The certified animal assisted therapy team consisted of a handler, who was also a member of the faculty at the center, and Boomer, a therapy dog certified by Delta Society/Pet Partners.

\section{Procedures}

After obtaining permission from parents and guardians for all of the children to participate in AAT during the therapy sessions, a single-case ABA (baseline-intervention-baseline) design, as described by Gast and Ledford was implemented to measure the number of spontaneous vocalizations BD produced with and without AAT. ${ }^{22}$ The Institutional Review Board determined that the 
case study was exempt from IRB review. The initial baseline (A) condition consisted of four sessions involving typical therapy procedures and group activities, without AAT. The intervention (B) condition consisted of four sessions that involved application of the AAT team. The second baseline (A) condition consisted of three sessions without the AAT team, during typical therapy procedures and activities. During the sessions for the intervention (B) condition, the team was brought into the room and introduced to the children. Children were given opportunities to interact with the dog, but were not required to do so. The handler, who is also a faculty member at the center, followed a prearranged protocol for the interaction, shown in the table below, which was similar to interactions conducted during other group and individual sessions at the clinic.

Table. Protocol for AAT handler

- Walk into the room with the dog and introduce the dog. Then sit on the floor with the dog.

- Demonstrate how to use the brushes (bath sponges with handles) to pet the dog. Have graduate student clinicians demonstrate brushing and offer brushes to the children.

- If children approach the dog, demonstrate how to pet him gently with their hands and allow them to pet the dog. Give verbal correction and demonstration if needed.

- If any child, including BD, does not approach the dog, allow the child to play in another area of the room with desired toys. During that time, a graduate student clinician will talk about what the child is doing and attempt to engage the child in play, according to the typical manner of interaction during the sessions. After a few minutes, ask any child who has not interacted with the dog if he/she would like to pet the dog.

- Talk about the dog and what the children are doing (e.g., "You're petting Boomer," "Boomer likes that," "Pet gently,"). Do not single out $\mathrm{BD}$, but do respond to $\mathrm{BD}$ or any other child's utterances or attempts to interact.

- After 15 minutes, a signal will be given to end the interaction. Gather the brushes and tell the children it is time for Boomer to leave, and leave the room.

Graduate master's level speech-language pathology student clinicians and faculty supervisors who all routinely interacted with the children and were familiar with typical procedures for the group sessions were instructed to interact with the children and to use the same therapeutic techniques (i.e., modeling words and phrases, commenting on the activity, scaffolding children's utterances) as used in the typical therapy (A) condition. In both conditions, this portion of the session (middle) was designed to be child-led, in the sense that intervention techniques centered around items and activities in which the children showed interest. In the (A) condition, this included a variety of age-appropriate toys and play activities. These same toys and activities were available in the (B) condition, with AAT incorporated as an additional stimulus and opportunity for engagement.

Data were collected live, using a Tap Counter application, by a single investigator, for 15-minute intervals each session. Data for all sessions were collected within the same 30-minute time frame (i.e., middle of the session). The dependent variable was the total number of spontaneous vocalizations BD produced in 15 minutes. The independent variable was the presence of and opportunity for interaction with a therapy dog.

\section{RESULTS}

The first purpose of the case study was to investigate the effect of AAT on the number of vocalizations produced by BD in a 15minute interval. During the four sessions in the first baseline $(A)$ condition, $B D$ produced $0,5,2$, and 0 vocalizations, respectively, during the 15-minute interval. During the intervention (B) condition, the number of vocalizations per 15-minute interval reflected a considerable increase, with 49,60,90, 94 vocalizations, respectively, for each of the four sessions. During the second baseline (A) condition, the number of vocalizations recorded during the 15-minute interval in the second baseline condition was 70, 100, and 63, respectively. Results are shown in Figure 1. 


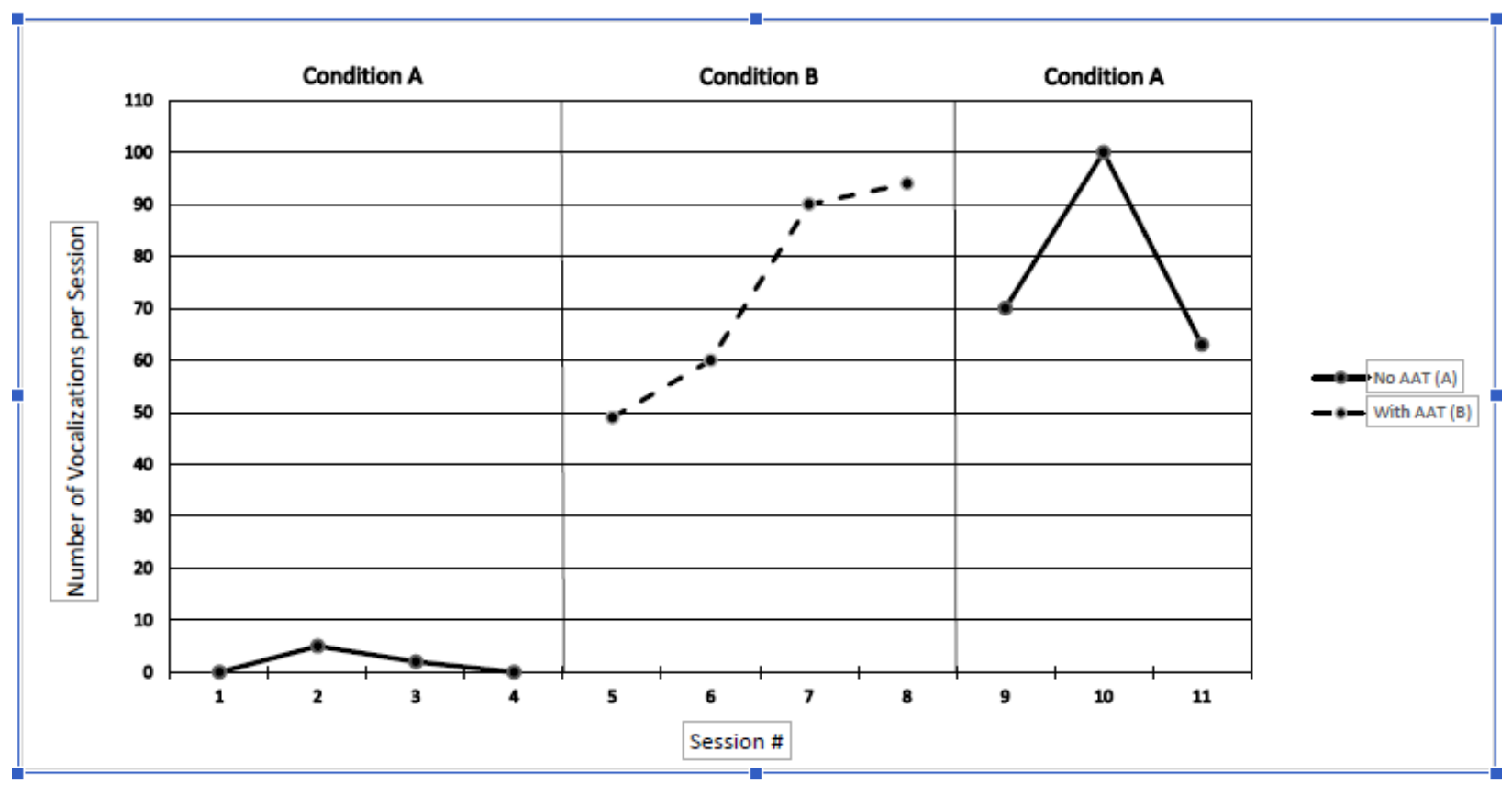

Figure 1. Vocalizations With and Without AAT

In addition to quantitative information concerning the number of vocalizations, qualitative observations were made about BD's response to $A A T$. One observation was related to the nature of the vocalizations $B D$ produced during the intervention $(B)$ and second baseline $(A)$ conditions. Not only did BD produced a higher number of spontaneous vocalizations that met the study criteria (see Methodology section) during the AAT sessions; he also produced recognizable word approximations related to the AAT activity (e.g., dog and bye-bye), a communication behavior that BD had not demonstrated during prior group sessions. The vocalizations $\mathrm{BD}$ produced during the intervention phase in this study were qualitatively different from phonation behaviors previously exhibited by the child in that they met one or more of the following descriptions: involved eye contact, had a clear referent, were accompanied by gestures/pointing, could clearly be interpreted as having specific intent based on context of the utterance, were word approximations, or were produced directly following a stimulus (contingent response).

Another observation concerned BD's attention to task. Prior to the case study, BD had consistently demonstrated limited attention to task, typically moving rapidly between items or activities of interest, and requiring maximal redirection to sustain attention to structured activities. During the first two sessions of the intervention phase, he demonstrated sustained attention to the therapy dog for more than five minutes, which was a longer duration compared to his sustained attention to any one activity during the same component (i.e., same time frame within the session) of group therapy sessions he had attended prior to initiation of this study. During the third and fourth sessions, BD did not show the same duration of sustained attention to the dog, but continued to return to the dog intermittently, sustaining attention to the dog for multiple 3 to 5 minute intervals. During all intervention (B) sessions, he produced vocalizations while paying attention to the dog.

The second purpose of the study was to evaluate AAT methods to determine optimal practices for AAT intervention involving young children with communication disorders. The researchers observed that the effect of AAT (increased vocalizations by BD) was evident within the first 5 to 10 minutes of each intervention session. After 10 minutes, some of the children in the group demonstrated decreased interest in the therapy dog, while others, including BD, demonstrated increased physical activity, including a greater frequency of sudden or unpredictable movements around the dog. For example, during the last five minutes of the third and fourth intervention session, BD attempted to ride the dog or lie down on top of him, or simply pushed or handled the dog roughly. The handler was able to respond by gently blocking movements that appeared forceful.

\section{DISCUSSION}

The hypothesis for the case study was that the number of vocalizations produced by BD in 15 minutes would be higher during sessions when AAT was used than during sessions when AAT was not used. Results supported this hypothesis. The number of vocalizations $\mathrm{BD}$ produced increased from an average of 1.75 during the four initial baseline sessions, to an average of 73.25 
during the four AAT sessions. This increase was interpreted as being non-reversible; that is, the number of vocalizations did not return to baseline. The non-reversibility of this result does not refute the hypothesis. Gast and Ledford noted that non-reversible behaviors often occur in single-case ABA designs involving behavioral interventions. ${ }^{22}$ The potential for generalization of behavior changes from the intervention (B) phase to subsequent baseline (A) phases is a characteristic inherent to the application of this design. From a clinical standpoint, the generalization of BD's increase in vocalizations to subsequent sessions was a positive outcome, because the primary goal of the group therapy program was to increase communication skills. The increase in BD's vocalizations that occurred with the introduction of AAT is consistent with the finding by LaFrance et al of increased verbal responses associated with AAT, and with overall positive effects on communication and motor behaviors found by other researchers. $7,11,14,20$ The incidental finding that BD's attention to tasks and interactions increased in the presence of the therapy dog was consistent with Heimlich, Limond et al, and Martin and Farnum. This incidental finding also provided support for the biocentric developmental model proposed by Sams et al.2,4,6,12

Another aspect of the change in BD's behavior that warrants discussion is the degree of change observed. Although BD was selected for the case study because he had demonstrated very limited expressive communication, the magnitude of the increase in vocalizations that occurred was unanticipated. During the (B) condition, BD produced sounds and word approximations (e.g., /da/ [dog] while pointing to the dog) that he had not produced during any prior group sessions; in fact, during prior sessions he had rarely made sounds at all. Because of this prior history of lack of vocalizations, also reflected in the low number of vocalizations noted during the baseline $(A)$ condition, the dependent variable was broadly defined to include a variety of vocalizations, regardless of whether or not the vocalizations were intelligible or if the linguistic function of the vocalization could be identified with certainty. Had BD produced recognizable words or approximations during the baseline (A) condition, the dependent variable could have been modified to incorporate a more specific, narrower operational definition of vocalizations (e.g., including only recognizable word approximations), which would have resulted in a lesser degree of change from baseline to intervention conditions. This decision to employ a broader operational definition when studying the intervention with $\mathrm{BD}$ is reflective of the process that can occur when setting treatment goals in clinical scenarios with individuals who have very limited language or pre-language skills: it is desirable to set very specific behavioral objectives in order to measure progress as accurately as possible; however, when there is no existing repertoire to build on, establishing a behavior (e.g., vocalizations) can be an appropriate goal initially; then, as communication behaviors become more sophisticated, treatment goals can be expressed and defined according to more detailed criteria and task hierarchies. It is not assumed that all children with severe communication disorders or other disabilities would demonstrate the same degree of response to AAT that was observed with BD. However, the results observed with BD do indicate that AAT can have a substantial impact on communication behaviors in some children with speech and language delays.

Regarding the second purpose of the study, determining optimal methods for AAT research with young children in a setting such as the one described, several considerations were identified for future studies. First, 15 minutes of AAT was long enough, and perhaps too long, for the sessions in this study. This consideration is based on the increase in unpredictable, sudden movements that $\mathrm{BD}$ and other children in the group demonstrated after 10 minutes. The dog was a certified therapy dog and was therefore trained to remain calm even when handled roughly, and the handler always maintained a priority of protecting the dog, so that the dog was not harmed in any way. However, as suggested by Burrows et al when designing AAT research studies, researchers should maintain awareness of factors in the environment or procedures that could potentially be stressful for the AAT team, and adjust the design and procedures to minimize stressors. ${ }^{19} \mathrm{It}$ is recommended that in future research similar to this case study (i.e., involving young children with severe disabilities), the intervention period should be limited to 10 minutes or less to avoid potential stress on both the handler and therapy dog. Prior to the sessions, the researcher and handler should discuss exactly how the handler and researchers will respond to any instances of challenging or aggressive behavior by child participants and establish criteria for discontinuing the AAT intervention if needed.

One limitation of this research was the scope of the study; the ABA design was applied to only a single participant. This limitation may be addressed in future research by considering other types of single-subject designs, such as multiple baselines, and/or increasing the number of participants. Limiting this study to a single participant did allow the researchers to widen the focus of the research to include the second purpose, which was to evaluate the AAT practices used in the study, with the intent to develop recommendations for best practices.

A second limitation was that a full transcript of BD's vocalizations was not recorded for further analysis. Because the dependent variable was the rate of vocalizations (number of vocalizations during a 15-minute interval), a count of vocalizations was chosen as the method for recording data. However, recording a transcript of the vocalizations would have allowed for further analysis of the vocalizations by the investigator and/or the reader in terms of message content, pragmatic functions, or other linguistic features. Recording and transcription of all vocalizations is recommended for future studies of a similar nature. 
By evaluating the AAT methods used with BD and the other children in the therapy group, and generating recommendations for AAT research practices, this case study addressed the call by other AAT researchers for research concerning the welfare of service and therapy animals. ${ }^{1,15,19}$ Careful planning is essential in any research with young children to ensure that the welfare of the child participants is protected; however, the use of AAT also warrants additional preparation and planning during the design stage to ensure that the welfare of the animal is equally protected. Findings from this case study also support the "proof of concept" described by O'Haire; that is, the overall trend seen in the literature of positive outcomes associated with AAT, despite the variability of research questions and methods. 11(p.1619) Although this case study included only one participant, these results suggest that AAT can be a valuable tool for speech-language pathologists when providing early intervention services.

\section{REFERENCES}

1. Nimer J, Lundahl B. Animal-assisted therapy: A meta-analysis. Anthrozoos. 2007;20(3):225-38. Retrieved from http://patastherapeutas.org/wp-content/uploads/2015/07/MetaAna\%CC\%81lise.pdf

2. Heimlich K. Animal-assisted therapy and the severely disabled child: A quantitative study. J Rehabil. Oct 2001;67(4):48-52.

3. Granger B, Kogan L, Fitchett J, Helmer K. A human-animal intervention team approach to animal-assisted therapy. Anthrozoos. 1998;11(3):172-6.

4. Limond JA, Bradshaw J, Cormack M. Behavior of children with learning disabilities interacting with a therapy dog. Anthrozoos. 1997;10(2-3):84-9. https://doi.org/10.2752/089279397787001139

5. Macauley B. Animal-assisted therapy for persons with aphasia: A pilot study. J Rehabil Res Dev. 2006;43(3):357-66. [PMID: 17041821]

6. Martin F, Farnum J. Animal-assisted therapy for children with pervasive developmental disorders. West J Nurs Res. 2002;24(6):657-70. [PMID: 12365766]

7. Nathanson D, deCastro D, Friend H, McMahon M. Effectiveness of short-term dolphin-assisted therapy for children with severe disabilities. Anthrozoos. 1997;10(2-3):90-100.

http://www.tandfonline.com/doi/abs/10.2752/089279397787001166

8. Cole K, Gawlinski A, Linquist R, Kirksey K. Animal-assisted therapy: the human-animal bond. AACN Clinical Issues. 2000;11(1):134-49. [PMID: 17962502]

9. Motomura N, Yagi T,Ohyama H. Animal assisted therapy for people with dementia. Psychogeriatric.2004;4:40-2. http://www.agatea.org/LMA/ECMAN003.pdf

10. Edwards N, Beck A. Animal-assisted therapy and nutrition in Alzheimer's Disease. West J Nurs Res. 2002:24(6):697712. [PMID 12365769]

11. O'Haire M. Animal-assisted intervention for autism spectrum disorder: A systematic literature review. J Autism Dev Disord. 2013;43:1606-22. [PMID: 23124442]

12. Sams M, Fortney E, Willenbring S. Occupational therapy incorporating animals for children with autism: A pilot investigation. Am J Occup Ther. 2006;60(3):266-74. [PMID: 16776394]

13. Velde B, Cipriani J, Fisher G. Resident and therapist views of animal-assisted therapy: Implications for occupational therapy practice. Australian Occup Ther J. 2005;52:43-50.

http://www.halsansnatur.se/images/media/velde\%20et\%20al.pdf

14. Macauley B, Guitierrez K. The effectiveness of hippotherapy for children with language-learning disabilities. Commun Disord Q. 2004;25(4):205-17. http://journals.sagepub.com/doi/pdf/10.1177/15257401040250040501 
15. Endenburgh N, van Lith $H$. The influence of animals on the development of children. Vet J. 2011;190(2):208-14. http://www.sciencedirect.com/science/article/pii/S1090023310004077

16. Haubenhofer $\mathrm{D}$, Kirchengast $\mathrm{S}$. Physiological arousal for companion dogs working with their owners in animal-assisted activities and animal-assisted therapy. J Appl Anim Welf Sci. 2006;9(2):165-72. [PMID 16956319]

17. Marinelli L, Normando S, Siliprandi C, Salvadoretti M, Mongillo P. Dog assisted interventions in a specialized centre and potential concerns for animal welfare. Vet Res Comm. 2009;33:S93-5.[PMID: 15372944]

18. Williamson C. Dolphin assisted therapy: Can swimming with dolphins be a suitable treatment? Dev Med Child Neurol. 2008;50(6):477. [PMID: 18489459] doi: 10.1111/j.1469-8749.2008.00477.x

19. Burrows $K$, Adams $C$, Millman J. Factors affecting behavior and welfare of service dogs for children with autism spectrum disorder. J Appl Anim Welf Sci. 2008;11(1):42-62. [PMID: 1844026]

20. LaFrance C, Garcia, Labreche J. The effect of a therapy dog on the communication skills of an adult with aphasia. J Comm Disord. 2007;40(3):215-24. [PMID: 16950329]

21. Stark R, Bernstein L, Demorest M. Vocal communication in the first 18 months of life. J Speech Lang Hear Res. 1993;36:548-58. [PMID: 8331912]

22. Gast D, Ledford J. Single Case Research Methodology: Applications in Special Education and Behavioral Sciences, 2e. New York, NY: Routledge; 2014. [PMID: 28218597] 